Revised November, 2000

\title{
The Pavlovian Response of Term Rates to Fed Announcements*
}

\begin{abstract}
The traditional view of the monetary transmission mechanism rests on the premise that the Federal Reserve (Fed) controls the level of the Federal funds rate via open market operations and the liquidity effect. By contrast, this paper argues that the Fed also manipulates the Federal funds rate via public disclosures of the new level of the Federal funds rate target and the "announcement effect." We define the announcement effect as the portion of interest rate movements associated with public statements on interest rate targets that do not require conventional open market operations for their support. This paper provides evidence on how the Fed uses the liquidity effect in conjunction with the announcement effect to execute monetary policy. In addition, it investigates the implications of the announcement effect on term structure behavior and the rational expectations hypothesis.
\end{abstract}

- JEL Classification Codes: E5, E4, C5

- Keywords: Liquidity Effect, Announcement Effect, Term Structure, Marked Point Process.

Selva Demiralp

Board of Governors of the Federal Reserve

Division of Monetary Affairs

Washington, DC 20551

E-mail: selva.demiralp@frb.gov

Oscar Jorda

U.C. Davis

Dept. of Economics

One Shields Ave.

Davis, CA 94596

e-mail: ojorda@ucdavis.edu

*The authors acknowledge many useful comments and suggestions from seminar participants at the Federal Reserve Bank of New York, Simon Frasier University, U.C. San Diego and U.C. Davis. We are particularly indebted to Jim Hamilton, Kevin Hoover, Kenneth Kuttner, and Daniel Thornton. All errors are our own responsibility. The opinions expressed are those of the authors and do not necessarily reflect the views of the Board of Governors of the Federal Reserve System or other members of its staff. 
Chairman Greenspan: "Look, the main issue here is that, as far as I am concerned, I would like us to be counted. We are the central bank and we are making a major move." Transcript of the Federal Open Market Committee Meeting, February 3-4, 1994.

\section{Introduction}

The Federal Open Market Committee Meeting (FOMC) held February 3-4, 1994 marked the first change in the Federal funds rate target since September 4, 1992. During that meeting, a fair amount of the discussion centered about the need to make intentions clear to the public. After all, rates had not been changed for close to two years and it had been five years since the Fed had last taken a tightening stance. The meeting concluded with the most important press release since Chairman Volcker announced the beginning of the now infamous nonborrowed reserves targeting experiment of the 1979-1982 period: The Federal funds rate target had been increased by 25 basis points. Since the February 3-4, 1994 meeting and up to present time, the Fed has continued the practice of publicly disclosing the new level of the Federal funds rate target immediately after each FOMC meeting.

In this paper we argue that this change in procedure has added a new policy tool to the Fed's arsenal: the announcement effect. The conventional view of the monetary transmission mechanism kicks off with the liquidity effect and the Fed's ability to manipulate short-term interest rates by actuating on a "monopolistically" held supply of bank reserves. However, we will argue that the financial and institutional evolution of the reserves market 
makes it possible for the Fed to exert moral suasion on the overnight rate. Therefore, the announcement effect reflects the Fed's ability to manipulate the overnight rate by publicly disclosing its desired level - an adjustment that takes place with a volume of open market operations much smaller than a liquidity effect would suggest. This is the essence of the Pavlovian response of the banking system we wish to explore.

Guthrie and Wright (2000) are perhaps the first to investigate a similar phenomenon which they term "open mouth operations." Although closely related to the announcement effect we want to investigate, there are fundamental differences worth pointing out. Their research centers on the ability of the Reserve Bank of New Zealand (RBNZ) to control the official cash rate (basically the overnight rate) without changing conventional policy instruments. Although there are no reserve requirements in New Zealand, banks hold reserves for settlement purposes and are free to trade any excess with each other. The central bank offers a lending facility that stands ready to supply an arbitrary amount of overnight settlement cash at a fixed rate, effectively capping the overnight rate. At the same time, excess settlement cash can be deposited with the RBNZ at a rate that acts as a floor to the overnight rate. The effective range at which overnight trades take place is therefore determined by the lending and deposit rates. Open mouth operations then occur whenever the central bank announces a new target for the official cash rate. The announcement itself constitutes a surprise to the market since the overnight rate typically trades at a level consistent with a monetary conditions index. Thus, the announcement of a new target rate constitutes a correction in expectations formulated by the RBNZ. 
Unlike the RBNZ (and other countries without reserve requirements such as the U.K., Sweden, Canada and Australia) the announcement of a new level for the Federal funds rate target does not necessarily constitute a surprise to the market. In fact, one can argue that these changes are widely anticipated. This feature constitutes a main avenue of departure with respect to Guthrie and Wright's open mouth operations since measuring the exogenous response to the announcement of a new target level requires that we model expectations of future target changes. The methodology we use to separate announcements into their expected and surprise components is a variant of the autoregressive conditional hazard (ACH) model proposed by Hamilton and Jordá (2000).

Open mouth operations occur because the RBNZ poses a credible threat: it controls the lending rate and the deposit rate and is ready to act on these rates to achieve the desired target level (Woodford, 2000 contains a lucid explanation of this monetary control mechanism which he terms a "channel system"). By contrast, the Fed does not pay interest rates on reserves (although the Fed compensates banks holding clearing balances in the form of credit for service fees) and although the discount window acts as a lending facility, for institutional reasons to be discussed below, it is rarely used by banks (not to mention that the discount rate is an administered rate typically set below the Federal funds rate). Therefore, the "credible threat" argument presented in Guthrie and Wright (2000) is of necessity more subtle in the U.S.: one needs to explain where is the necessary slack built into the reserves market to absorb the announcement effect.

Other efforts dedicated to measuring open mouth operations in the U.S. are probably 
motivated by the observation that the presence of an announcement effect complicates the measurement of the liquidity effect and could explain the "vanishing liquidity effect" reported by Pagan and Robertson (1995) and Christiano (1995). Thornton (2000) investigates the reaction of the Federal funds rate and other T-Bill rates to changes in the target using daily data. However, there is little evidence supporting the liquidity effect over the announcement effect or vice versa, forcing Thornton to conclude that changes in the target reflect the Fed's reaction to market forces rather than policy initiative. This argument underscores once again the importance of measuring the exogenous component of target changes if one is to properly measure the significance of the announcement effect.

This paper is structured as follows. In section 2 we discuss recent institutional developments at the Federal Reserve that guide the empirical analysis executed below. Section 3 illustrates the intricacies of the reserves market and the identifying assumptions that permit us to calculate the relative magnitude of the liquidity effect and the announcement effect. Section 4 studies the manner in which target announcements are incorporated into the yield curve by decomposing announcements into their anticipated and unanticipated components with the ACH model. Section 5 reports the empirical tests of the effect of unanticipated announcements on the term structure. Section 6 presents our conclusions.

\section{Recent Developments in the Reserves Market}

This paper concentrates on the period March 1984 to March 1999 during which the Fed has maintained fairly stable operating procedures. After the 1979-1982 nonborrowed reserves 
targeting experiment, the Fed moved to a borrowed reserves target. According to Meulendyke (1998): "The procedures allowed a much smaller degree of variation in the funds rate than the nonborrowed reserves procedures that had preceded it." (p. 53), although "[...] policy intentions were less transparent than with direct Federal funds rate targeting." (p. 53). The informal move away from borrowed reserves targeting into direct Federal funds rate targeting was speeded by the stock market crash in October 19, 1987 - a practice that has been maintained through the 1990's. Furthermore, Hamilton and Jordá (2000) argue that a borrowed reserves target can be viewed as a Federal funds rate target when demand for reserves is rather stable.

With the backdrop of the Federal funds rate targeting regime, a significant episode has influenced day to day operations at the Fed: the introduction of "retail sweep programs" in January, 1994. According to Anderson and Raasche (2000), the essence of these programs is computer software that dynamically reclassifies customer deposits between transaction deposits (subject to reserve requirements) and money market accounts (non-transaction deposits that are not subject to reserve requirements). Thus, in addition to cuts in reserve requirements in 1990 and 1992, required reserves have declined from about $\$ 60$ billion in 1994 to below $\$ 45$ billion in 1999 (see figure 1 ). This decline is mirrored by the change in the relative weight of non-transaction deposits to transaction deposits: from a 2:1 ratio in 1994 to a 4:1 ratio in 1999 (see figure 2).

Banks hold deposits at the Federal Reserve primarily for three reasons: (1) to meet statutory reserve requirements; (2) to clear day to day interbank transactions mediated by 
the Fed (clearing balances); and (3) to prevent shortfalls in the requirement or to prevent overdrafts on clearing balances (excess reserves). Banks do not receive interest payments for balances maintained to meet reserve requirements or for excess reserves but as Anderson and Raasche (2000) point out, deposit-sweep activity has reduced the economic burden of statutory reserve requirements essentially to zero. Clearing balances on the other hand, are remunerated with credit toward the transaction fees charged by the Fed for services provided. Therefore, although the Fed still maintains unremunerated reserve requirements, their practice is much closer to that of the central banks in Australia, New Zealand, Canada and the U.K., which do not have reserve requirements.

The volatile nature of daily interbank transactions (the flow of payments and receipts is around 30 times greater than a bank's overnight reserve balance according to Furfine, 2000) overshadows the uncertainty on reserve requirements and motivates the banks' desire to maintain excess reserves. Associated with this demand for excess reserves is the banks' reluctance to use the discount window (even if the discount rate is typically well below the Federal funds rate): although extended credit reached volumes in excess of $\$ 7$ billion at the height of the Savings \& Loans crisis in the mid-eighties, the reputation costs of borrowing from the Fed have essentially reduced this volume to zero in the nineties.

The Fed has adapted its institutional practices to this new state of affairs in several ways. Beginning 1997, daily open market operations were moved back from 11:30 am to 10:30 am and more recently to 9:30 am, with long-term repurchase agreements being arranged as early as 8:30 am. While the Desk normally arranges temporary open market operations no 
more than once a day, "The Desk always was prepared to depart from its usual practices as circumstances warranted" (Federal Reserve Bank of New York, Markets Group, 1999, p.16), thus allowing the Desk the flexibility to enter the market more than once per day. In August 18, 1998 the Fed dropped the contemporaneous reserve accounting in favor of lagged reserve accounting, thus drastically reducing the ambiguity on the reserve need during the final days of the maintenance period. Along with the Fed's disposition to accommodate reserve demand shocks documented in Strongin (1995) among others, these developments suggest that the traditional view of the reserves market depicted in earlier papers needs to be reformulated.

In addition to the institutional considerations discussed above, the key element of our analysis is to detect when banks started reading the Fed's intentions with clarity. The obvious institutional break point is the February 3-4, 1994 FOMC meeting which established the practice of publicly announcing the new level of the fed funds target. However, we believe banks had accurate information on the timing of target changes well before then. In particular, Hamilton and Jordá (2000) discuss a break in the manner policy was conducted in November 22, 1989. This sometimes called "Thanksgiving effect" took place when the Fed added reserves at a time when the fed funds rate was below its $8 \frac{1}{2} \%$ target. The market interpreted this as a signal that the target had been lowered and a Fed policy change was announced in the business press (Wall Street Journal, 11/24/89, p. 2; 11/28/89, p. 1). In fact, the Fed had not changed the target but had added reserves due to an erroneous borrowed reserves forecast (further details on this event are contained in the above reference). 
Figure 3 displays the daily spread between the prime rate $^{1}$ and the Federal funds rate target and clearly illustrates the Thanksgiving effect. After November 22, 1989 there is virtually no uncertainty in reading Fed moves: banks adjusted the prime rate, often within the same day of a target change, and with certainty, during the span of a maintenance period. We are therefore persuaded that the 1989 breakpoint constitutes a reasonable alternative to the 1994 breakpoint. In addition, there are several advantages to splitting the sample in 1989. The Federal funds rate futures market, which will provide us with a way to calculate market expectations via futures prices, was created in 1989. Moreover, the 1989 breakpoint affords us a few more target changes (somewhat more scarce after 1994) which help us identify the announcement effect with more clarity.

The next section combines all the elements discussed above to formulate a model for the reserves market and an empirical strategy that will permit us to measure the announcement effect.

\section{Identification of Announcement Effects}

The standard assumption in most models of the monetary transmission mechanism is that the Fed influences the Federal funds rate via open market operations. In fact, "To implement the FOMC's policy instructions, the open market area seeks to manage reserve levels of depository institutions in a way that will encourage the Federal funds rate to trade around the level agreed to by the Committee," (Meulendyke, 1998, p. 139). Under this assumption,

\footnotetext{
1 The prime rate is conventionally defined as the rate banks charge on loans to their preferred customers. The role of the prime rate has changed substantially in recent times although it is still routinely used as an index rate.
} 
innovations on a monetary aggregate controlled by the Fed (under suitable identification assumptions) are often equated to policy innovations. However, as a matter of actual practice, Strongin (1995) points out that the Federal Reserve accommodates innovations in the banking system's demand for reserves. Therefore, failing to account for this policy of accommodation may mislead one to believe these innovations are policy driven.

Existing models of the reserves market, such as those proposed by Strongin (1995) and Bernanke and Mihov (1998a, b) emphasize the Fed's willingness to reconcile demand innovations by appropriately choosing the mix of nonborrowed reserves and borrowed reserves it chooses to supply. A brief exploration of Strongin's (1995) model will help us highlight the main points. In its innovation form (i.e., the residuals from the reduced form representation of the corresponding VAR), the model can be summarized by the following relations:

$$
\begin{aligned}
& u_{n b r}=v_{s}+\phi v_{d} \\
& u_{b r}=-v_{s}+(1-\phi) v_{d} \\
& u_{t r}=v_{d} \\
& u_{f f}=\lambda v_{s}+\pi v_{d}+v_{f f}
\end{aligned}
$$

The $u_{i}$, for $i=n b r ; b r ; t r ; f f$ refer to the reduced form innovations for nonborrowed reserves, borrowed reserves, total reserves, and the Federal funds rate respectively. $v_{s}$ represents the policy shock, $v_{d}$ represents a reserve demand shock, and $v_{f f}$ represents a fed funds innovation. $\phi$ describes how reserve demand accommodations are split between nonborrowed reserves and 
borrowed reserves.

Under this set up, the assumption that a positive supply innovation in nonborrowed reserves is exactly offset by a reduction in borrowed reserves guarantees that innovations in total reserves represent demand innovations only. Thus, the identification of supply shocks, $v_{s}$, can be obtained as the orthogonal component of nonborrowed reserves innovations, $u_{n b r}$, to total reserve innovations, $u_{t r}$, or in the common nomenclature of the VAR literature, by choosing a Wold causal ordering that places total reserves ahead of nonborrowed reserves, with the federal funds rate ordered last. The liquidity effect can then be easily described by the response of the fed funds rate to innovations in nonborrowed reserves.

This view of the reserves market assumes that demand for reserves is rather inelastic and that given a sufficient reserve shortfall, banks would then use the discount window. However, the institutional discussion that preceded this section suggests that the role played by the discount window has been quite minimal in the nineties. In addition, the assumption that the demand for reserves is inelastic (even in the short-run) may be less realistic with the availability of the "retail sweep programs" discussed above. Therefore, consider generalizing Strongin's model by assuming that banks are willing to absorb supply and demand innovations, at least partially, by modifying the level of excess reserves they hold. We do not make a distinction between active management of required reserves and an actual choice of excess reserve holdings since excess reserves are computed as the difference between total reserves and required reserves. Thus, the expression for the innovations in excess reserves becomes, 


$$
u_{e r}=\gamma v_{s}-\psi v_{d}
$$

where $\gamma$ represents the portion of a positive supply innovation absorbed by a countervailing increase in excess reserves, and $\psi$ represents the portion of a demand shock buffered by reducing the level of excess reserves rather than by going to the discount window. Therefore, the model represented in (1) can be easily generalized to:

$$
\begin{aligned}
& u_{n b r}=v_{s}+\phi(1-\psi) v_{d} \\
& u_{b r}=-(1-\gamma) v_{s}+(1-\phi)(1-\psi) v_{d} \\
& u_{t r}=\gamma v_{s}+(1-\psi) v_{d}
\end{aligned}
$$

so that $u_{t r}$ no longer contains demand innovations exclusively. Notice that by setting $\gamma=$ $\psi=0$, one recovers Strongin's original formulation in (1), while setting $\gamma=\psi=1$, would correspond to the extreme case in which banks refuse to use the discount window and use their management of excess reserves instead. From equations (2) and (3) and noting that total reserves are the sum of required reserves and excess reserves, we have

$$
\begin{aligned}
& u_{t r}=\gamma v_{s}+(1-\psi) v_{d} \\
& -u_{e r}=-\gamma v_{s}+\psi v_{d} \\
& u_{r r}=v_{d}
\end{aligned}
$$

so that it is required reserves (instead of total reserves) the component that contains demand innovations exclusively. Therefore, by replacing total reserves with required reserves in the 
original formulation proposed in (1), the liquidity effect can be depicted with the impulse response of the fed funds rate to a shock in nonborrowed reserves just the same.

In combination with the liquidity effect, we argue that the Fed uses the announcement effect. To measure the relative importance of each of these effects, we base our empirical strategy on the following observation. In an environment in which there is no announcement effect, the magnitude of the liquidity effect that one can compute with a VAR based on expression (4) is invariant to the inclusion or exclusion of dates in which the target was changed. By contrast, if the announcement effect is truly prevalent, one should expect the liquidity effect to be more pronounced precisely during dates in which the target was changed. The rationale for this last assertion is that small movements in nonborrowed reserves (perhaps associated with the direction of policy, perhaps associated to accommodation of expectations) will be related to large movements in the fed funds rate that are caused by the unobservable announcement effect. Consequently, estimating a VAR over a sample that includes dates of target changes will bias the results toward finding a stronger liquidity effect than there really is. The empirical strategy that we pursue exploits this bias.

\subsection{Empirical Evidence}

We analyze the reserves market at a bi-weekly, maintenance period frequency (i.e., bi-weekly starting Thursday of the first week of the maintenance period and ending settlement Wednesday of the second week) over two sample periods: March 25, 1984 to November 1, 1989 and November 15, 1989 to March 24, 1999. For each of these two sample periods, we estimate a structural VAR based on the model in (4) and therefore containing the following variables: 
required reserves normalized by lagged total reserves (RRX); nonborrowed reserves normalized by lagged total reserves (NBRX); and the Federal funds rate (FF). The normalization by lagged total reserves is standard in this literature and is aimed at preserving the linear relations described in (1) and (4) while filtering for any long term behavior in reserves. The Wold causal ordering is that in which we report the variables and is based on model (4).

For each sample period, we estimate two VARs. The first includes all observations in the sample out of which we compute the impulse response of the fed funds rate to shocks in normalized nonborrowed reserves and the impulse response of normalized nonborrowed reserves to its own shock. Then we estimate a flexible two-state VAR that allows the dynamic behavior of the VAR to be possibly different during periods when the target was changed. There are comparatively few target changes relative to the number of observations thus causing the dynamic estimates during target periods to be imprecise. By the same token, the dynamics of the model on periods in which the target was not changed are quite precisely estimated and can be directly used to compare to the impulse response functions of the full sample version VAR. All estimated VARs have a lag length of 4 which afforded sufficient degrees of freedom for estimation while providing sufficient safeguard against left over autocorrelation.

Figure 4 reports the results of this experiment. The left column corresponds to the pre-1989 sample while the right column corresponds to the post-1989 sample. The top row displays the response of the fed funds rate to shocks in nonborrowed reserves (labeled "liquidity effect") while the bottom row displays the behavior of nonborrowed reserves to 
its own shocks. The one standard deviation error bands that we report correspond to those of the single state VAR and are reported to give a sense of the significance of the difference in the responses between the single state and the two-state versions of the VAR. The label "1-state VAR" in the graphs refers to the single state VAR impulse responses while the label "2-state VAR" corresponds to the impulse responses of the two-state VAR during periods when the target was not modified.

The results are quite striking. In the pre-1989 sample, the full sample "liquidity effect" impulse response has the familiar shape found in other papers: a positive shock to supply innovations in nonborrowed reserves causes the fed funds rate to drop significantly. This liquidity effect response is even more pronounced for the two-state VAR although a look at the own response of nonborrowed reserves reveals that this may be a consequence of the higher persistence that NBRX shocks display in this case. The contrast with the post-1989 sample is quite remarkable. As we had anticipated, the "liquidity effect" measured by the two-state VAR is significantly more tenuous than the full sample response while there is no appreciable difference in the behavior of nonborrowed reserves between the full sample and the two-state responses to its own shock. The significant gap between the two liquidity measures that we report in the post 1989 supports our hypothesis that since 1989 there has been an announcement effect at play. The next sections explore the effect that this announcement has had on term rates. 


\section{Forecasting Changes in the Target}

The evidence reported in the previous section indicates that a measurable and sizeable announcement effect exists in the reserves market. The question we explore in the remainder of the paper is whether there is a similar effect in the transmission of monetary policy on term rates, a much more important link from a policy-making point of view. The approach we pursue is based on studying the behavior of the Federal funds rate target in a manner similar to the approach followed by Cook and Hahn (1989), Thornton (2000) and Kuttner (2000). In particular, in order to measure the effect of new target announcements on term rates, we need to decompose target changes into their anticipated/endogenous and unanticipated/exogenous components. ${ }^{2}$ In principle, one could exploit data from the Federal funds rate futures market to infer market expectations on future target changes using the approach advanced in Kuttner (2000). However, this market began operating in earnest in 1989, leaving us unassisted in calculating what happened prior to 1989. The methodology that we will use instead is based on the autoregressive conditional hazard $(\mathrm{ACH})$ model which produces forecasts of the timing of announcements, and an ordered probit (OP) model that forecasts the magnitude of these discrete changes.

The time series of target changes has unusual statistical properties and is typically referred to as a marked point processes in the statistics literature. First, the target is changed irregularly in time, that is, we are uncertain about when the target will be changed next

\footnotetext{
${ }^{2}$ We do not take the words anticipated and endogenous to be synonyms. Rather, we wish to highlight that the mechanics of calculating the endogenous component of a target change will consist on coming up with the best forecast of a target change conditional on all available information, even that knowledge that would suggest an endogenous response on the Fed's behalf.
} 
given information available today. Second, when the target is changed, it is typically in discrete increments of 25 basis points rather than in a continuous manner. The process describing when events take place in time is called a point process. The value that the point process takes at each event time is called the mark. These two characteristics would typically require a dynamic model for limited dependent variables which usually involves either difficult numerical integrations (for example, Eichengreen, Watson and Grossman, 1985; Davutyan and Parke, 1995) or Monte Carlo Markov chain simulations (see McCulloch and Rossi, 1994; Dueker, 1998). Instead, we will adopt the approach proposed by Hamilton and Jordá (2000) which only requires conventional maximization of the likelihood function by numerical techniques.

In particular, let $x_{t}=0$ if there is no change in the target during maintenance period $t$, and $x_{t}=1$ if there is a change - thus, $x_{t}$ describes the process for the points. Let $y_{t}$ be the magnitude of a target change if one occurs at time $t$ - the marks. Let $z_{t}$ denote a vector of exogenous variables that may affect the Fed's decision to change the target, such as production, prices, unemployment, and so on. Let $\mathcal{Y}_{t}$ denote the information set at time $t$. Our task is to model the joint probability distribution of $x_{t}$ and $y_{t}$, conditional on the past. Without loss of generality, the corresponding log-likelihood can be factored into

$$
\sum_{t=1}^{T} \log f\left(x_{t}, y_{t} \mid \mathcal{Y}_{t-1}, \theta_{1}, \theta_{2}\right)=L_{1}\left(\theta_{1}\right)+L_{2}\left(\theta_{2}\right)
$$

where 


$$
\begin{aligned}
& L_{1}\left(\theta_{1}\right)=\sum_{t=1}^{T} \log g\left(x_{t} \mid \mathcal{Y}_{t-1}, \theta_{1}\right) \\
& L_{2}\left(\theta_{2}\right)=\sum_{t=1}^{T} \log q\left(y_{t} \mid x_{t}, \mathcal{Y}_{t-1}, \theta_{2}\right)
\end{aligned}
$$

If $\theta_{1}$ and $\theta_{2}$ have no parameters in common, then maximization of (5) is equivalent to maximization of (6) and (7) separately. If this condition is not met, the parameter estimates will not be efficient but will remain unbiased. The next subsections discuss in turn the models for $L_{1}\left(\theta_{1}\right)$, and $L_{2}\left(\theta_{2}\right)$, namely the $\mathrm{ACH}$ model, and the OP model respectively.

\subsection{The Autoregressive Conditional Hazard Model (ACH)}

The ACH model seeks an answer to the following question: What is the probability that during the next maintenance period, the target will be changed, conditional on information available today? Denote this probability by $h_{t}$, typically referred to as the hazard in the duration literature. Then, $h_{t}=P\left(x_{t}=1 \mid \mathcal{Y}_{t-1}\right)$. In addition, we define the following auxiliary variables. Let $\left\{w_{1 t}\right\}, t=1,2, \ldots, T$ be a sequence that, for any date $t$ records the date of the most recent change in the target as of time $t$,

$$
w_{1 t}=t x_{t}+\left(1-x_{t}\right) w_{1, t-1} \quad \text { for } t=1,2, \ldots, T
$$

so that $w_{1 t}=t$ if the target changes on date $t$ and $w_{1 \tau}$ stays at $t$ for subsequent weeks $\tau$ until a new target change. In general, let $w_{j t}$ be the date of the $j^{\text {th }}$ most recent target change as of date $t$ : 


$$
w_{j t}=x_{t} w_{j-1, t-1}+\left(1-x_{t}\right) w_{j, t-1} \quad \text { for } j=2,3, \ldots \text { and } t=1,2, \ldots, T
$$

Using this notation, $w_{1, t-1}-w_{2, t-1}$ corresponds to the length of the duration between the most recent two target changes as of date $t-1$. In general, the duration between the $j^{\text {th }}$ and the $(j+1)^{t h}$ most recent target changes is $u_{j, t-1}=w_{j, t-1}-w_{j+1, t-1}$.

Going back to the hazard rate, note that if the only information contained in $\mathcal{Y}_{t-1}$ were the dates of previous target changes, the hazard rate $h_{t}$ would not change until the next target change. Let $\psi_{t}$ denote the expected length of time until the next change, then

$$
\psi_{t}=\sum_{j=1}^{\infty} j\left(1-h_{t}\right)^{j-1} h_{t}=\frac{1}{h_{t}}
$$

It is natural to generalize expression (10) by allowing $\psi_{t}$ to have a traditional linear time series representation for the conditional first moment and to incorporate the effects of exogenous variables, linearly. In an expression similar to that adopted in Hamilton and Jordá (2000),

$$
\left\{\begin{array}{c}
h_{t}=\left(\psi_{t}+\delta^{\prime} z_{t-1}\right)^{-1} \\
\psi_{t}=\omega+\sum_{j=1}^{p} \beta_{j} \psi_{w_{j, t-1}}+\sum_{j=1}^{q} \theta_{j} u_{j, t-1}
\end{array}\right.
$$

where the denominator is appropriately constrained to ensure that it is positive and $h_{t} \in$ $[0,1]$.

The modification that we propose here is inspired by the duration literature and in particular, by the proportional hazards model. We have experimented with the following 
alternative specification for the $\mathrm{ACH}(\mathrm{p}, \mathrm{q})$ model,

$$
\left\{\begin{array}{c}
h_{t}=\left[1+\exp \left(\lambda_{t}\right)\right]^{-1} \\
\lambda_{t}=\omega+\sum_{j=1}^{p} \beta_{j} \lambda_{w_{j, t-1}}+\sum_{j=1}^{q} \theta_{j} u_{j, t-1}+\delta^{\prime} z_{t-1}
\end{array}\right.
$$

This particular parametrization of the model has the advantage of leaving the parameter space unconstrained which simplifies the computations. However, note that the conditional expected duration now becomes $\exp \left(\lambda_{t}\right)$ rather than $\psi_{t}$. The likelihood associated with either expression (11) or (12) is simply

$$
L_{1}\left(\theta_{1}\right)=\sum_{t=1}^{T}\left\{x_{t} \log \left(h_{t}\right)+\left(1-x_{t}\right) \log \left(1-h_{t}\right)\right\}
$$

which is the specific form that corresponds to the log-marginal likelihood in expression (6) and which can be maximized numerically with respect to the vector of population parameters by standard procedures.

\subsection{An Ordered Probit (OP) Model for the Marks}

The discrete nature of target changes suggests the use of an ordered response model, similar in spirit to that in Hausman, Lo and Mackinlay (1992). Target changes typically occur in discrete increments of 25 basis points though some changes as small as 6.25 basis points were sometimes observed prior to 1990. Given that our time-unit is the maintenance period, most target changes belong to the following categories, $y_{i}=\{-0.50,-0.25,0,0.25,0.50\}$, with only a few other changes being reclassified to fit the preceding categories (we refer the 
reader to Table 2 for specifics). The 0 category is included to collect a few small changes of 6.25 basis points in size.

It is easiest to describe the model by indexing observations by events $i$, rather than dates, $t$ since target changes only occur at event dates. Consequently, $i=1$ corresponds to the first target change, $i=2$ to the second and $i=N$ to the last. Let $y_{i}$ denote the magnitude of the $i^{\text {th }}$ target change and $\mathbf{v}_{i}$ denote a vector of variables observed the maintenance period prior to the $i^{\text {th }}$ target change that may have influenced the Fed's decision on how much to change the target. We hypothesize the existence of an unobserved latent variable, $y_{i}^{*}$ such that,

$$
y_{i}^{*}=\mathbf{v}_{i}^{\prime} \pi+\varepsilon_{i} \quad \text { where } \varepsilon_{i} \mid \mathbf{v}_{i} \sim \text { i.i.d. } N(0,1)
$$

Then, the observed series of target changes is related to the unobserved latent process as follows,

$$
y_{i}=\left\{\begin{array}{lll}
-0.50 & \text { if } & y_{i}^{*} \in\left(-\infty, c_{1}\right] \\
-0.25 & \text { if } & y_{i}^{*} \in\left(c_{1}, c_{2}\right] \\
\ldots & \ldots & \ldots \\
0.50 & \text { if } & y_{i}^{*} \in\left[c_{4}, \infty\right)
\end{array}\right.
$$

where $c_{1}<c_{2}<c_{3}<c_{4}$. Therefore, the probability that the target will change by, say -0.25 , is given by

$$
P\left(y_{i}=-0.25 \mid \mathbf{v}_{i}\right)=P\left(c_{1}<\mathbf{v}_{i}^{\prime} \pi+\varepsilon_{i} \leq c_{2}\right)
$$


or, if we denote the standard normal cumulative distribution by $\Phi(z)$,

$$
\begin{aligned}
& P\left(y_{i}=-0.5 \mid \mathbf{v}_{i}\right) \quad=\Phi\left(c_{1}-\mathbf{v}_{i}^{\prime} \pi\right) \\
& P\left(y_{i}=-0.25 \mid \mathbf{v}_{i}\right)=\Phi\left(c_{2}-\mathbf{v}_{i}^{\prime} \pi\right)-\Phi\left(c_{1}-\mathbf{v}_{i}^{\prime} \pi\right) \\
& \begin{array}{lll}
\ldots & \ldots & \ldots
\end{array} \\
& P\left(y_{i}=0.5 \mid \mathbf{v}_{i}\right) \quad=1-\Phi\left(c_{4}-\mathbf{v}_{i}^{\prime} \pi\right)
\end{aligned}
$$

from which the log-likelihood of the model can be trivially expressed and maximized by conventional procedures with respect to the parameters of interest.

\subsection{Empirical Estimates}

The estimates of the $\mathrm{ACH}$ and $\mathrm{OP}$ models are based on the Federal funds rate target data reported in Rudebusch (1995) and updated from the Federal Reserve Bank of New York's web-site. The model is estimated over two subsamples discussed in the preceding sections: March 25, 1984 to November 1, 1989 and November 15, 1989 to March 24, 1999.

For each subsample, we considered a number of explanatory variables to try to predict the timing of changes in the target. These variables can be classified into four broad categories: (1) variables reflecting the state of the macroeconomy, such as inflation, unemployment, output growth, and the budget deficit; (2) monetary and financial aggregates, such as M2 growth, long term rates an the spread between the Fed funds rate and the 6 month T-Bill rate; (3) reserve market variables designed to capture any endogenous response to demand for reserves, such as the ratio of excess reserves to total reserves (ERX), the ratio of nonborrowed reserves to total reserves (NBRX) and the ratio of borrowed reserves to total reserves (BRX); 
and (4) variables of an operational nature, such as the dates of FOMC meetings, the time elapsed between a target change and the subsequent change in the prime rate, and the spread between the target and the discount rate. Table 1 reports the maximum likelihood estimates of the final ACH models for each subsample.

The estimates of the selected $\mathrm{ACH}(1,1)$ model suggest persistent serial correlation in the hazard for the first subsample, with $\theta+\beta=0.82$, somewhat less for the second subsample, with $\theta+\beta=0.54$. The variable $F O M C_{t}$ is a dummy variable that takes the value of 1 if there was an FOMC meeting at time $t$, and is 0 otherwise. The negative and significant coefficients associated with this variable indicate that the probability of target change rises significantly when the FOMC meets, a natural result. The variable $|F F R-T B 6|$ denotes the absolute value of the spread between the effective funds rate and the 6 month T-Bill. Large deviations of this spread from its average value also increase the probability of a target change. The variable Inflation Rate is based on the consumer price index and has the expected sign, large fluctuations in inflation prompt the Fed to act. The variable Duration $P R$ measures the time elapsed between the last two changes in the prime rate. These results are broadly consistent with what one would expect would cause the Fed to change the target.

In addition to model fit, we also explored the model's forecasting performance. The ACH produces forecasts of the probability that, conditional on information during the current maintenance period, the Fed will choose to change the target in the following maintenance period. We termed this probability as the hazard and we denote its forecast by $\widehat{h}_{t}$. On the basis of this probability forecast, one can construct the series of predicted changes, $\widehat{x}_{t}$ by 
comparing $\widehat{h}_{t}$ to the average hazard over the period, $\bar{h}$ as follows,

$$
\widehat{x}_{t}=\left\{\begin{array}{lll}
0 & \text { if } & \widehat{h}_{t}<\bar{h} \\
1 & \text { if } & \widehat{h}_{t} \geq \bar{h}
\end{array}\right.
$$

The statistics literature provides two conventional measures to gauge the model's performance: specificity and sensitivity. Specificity measures the proportion of events (i.e. $\left.x_{t}=1\right)$ that were properly forecasted $\left(\widehat{x}_{t}=1\right)$ while sensitivity measures the proportion of non-events $\left(x_{t}=0\right)$ properly forecasted $\left(\widehat{x}_{t}=0\right)$. As an illustration, had we chosen the forecast: " $\widehat{x}=1$ for all $t$, " our specificity measure would have scored a perfect $100 \%$ while our sensitivity measure would have scored a disastrous $0 \%$. The specificity and sensitivity values attained by the $\mathrm{ACH}$ models are quite well balanced and relatively high. For the first subsample the values are $74 \%$ and $55 \%$ respectively and for the second subsample $54 \%$ and $67 \%$ respectively.

Kuttner (2000) provides an alternative manner with which to construct the market's expectation of a target change. Based on the prices of futures contracts, surprises on the Federal funds rate target can be constructed as,

$$
\widetilde{\varepsilon}_{\tau}=\frac{m_{s}}{m_{s}-\tau}\left(f_{s, \tau}^{0}-f_{s, \tau-1}^{0}\right)
$$

for all but the first and last days of the month. $\widetilde{\varepsilon}_{\tau}$ denotes the surprise component of a target change, $m_{s}$ denotes the number of days in month $s, \tau$ is the day of the month in which the target is changed and $f_{s, \tau}^{0}$ denotes the spot-month futures rate at day $\tau$ of month $s$ over which the average effective overnight funds rate is computed to price the contract (we refer 
the reader to the original source for a more detailed discussion). The expected component of a target change can then be calculated as $\Delta \widetilde{r}_{\tau}^{e}=\Delta \widetilde{r}_{\tau}-\widetilde{\varepsilon}_{\tau}$.

This last magnitude can be used in a simple logit model to calculate the conditional probability of a target change given today's futures contract prices. Note that this is a oneday ahead forecast of a target change rather than a maintenance-period ahead forecast (which is what the ACH model produces). With these alternative hazard forecasts, we constructed event forecasts ( $\widehat{x}_{t}$ in our notation) and checked the market's ability to foresee changes in the target. Table 3 reports the logit estimates as well as the specificity and sensitivity values, which are $89 \%$ and $81 \%$ respectively - an indication of the market's reasonable ability to read the Fed's intentions one day in advance.

Table 2 reports the results for the OP model. The amount of borrowing at the discount window (measured by $B R X$, the ratio of borrowed reserves to lagged total reserves) as well as the spread between the Fed funds rate and the 6-month T-Bill, prompt the Fed to lower the target. On the other hand, the spread between the target and the discount rate $(S F F R T D R)$, the spread between the prime rate and the target $(S P R F F R T)$ and the duration between a target change and a discount rate change $(D F F R T D R)$, contribute to target increases. These results are also broadly consistent with the way we would expect the Fed to behave. 


\section{Announcement Effects on the Term Structure}

This section investigates the effect that exogenous changes in the Federal funds rate target had before and after the 1989 break period on term rates. The ACH/OP methodology of the previous section allows us to easily compute expectations of target changes as,

$$
\begin{aligned}
\Delta \widetilde{r}_{t}^{e}= & \left(1-\widehat{h}_{t}\right) \times 0+ \\
& \widehat{h}_{t} \times\left[-0.50 \cdot \widehat{p}_{-0.50}-0.25 \cdot \widehat{p}_{-0.25}+\ldots+0.50 \cdot \widehat{p}_{0.50}\right]
\end{aligned}
$$

where $\widehat{h}_{t}$ is the ACH forecast of next period's hazard and $\widehat{p}_{j}$ is the OP forecast of the probability of a $j$ percentage change, given that the target will be changed. In addition, we will use the $\Delta \widetilde{r}_{t}^{e}$ that can be calculated following Kuttner (2000) in the manner described in section 4 as a robustness check of our results for the post-1989 sample.

A natural way to measure the impact of target announcements on term rates is by running the following simple regression,

$$
\Delta R_{t}=\left[\alpha_{1}+\beta_{1} \Delta \widetilde{r}_{t}^{e}+\gamma_{1} \widetilde{\varepsilon}_{t}\right] d_{t}^{C}+\left[\alpha_{2}+\beta_{2} \Delta \widetilde{r}_{t}^{e}+\gamma_{2} \widetilde{\varepsilon}_{t}\right]\left(1-d_{t}^{C}\right)+u_{t}
$$

where $R_{t}$ denotes 3-month, 6-month, 1-year, 2-year, 5-year, 10-year and 30-year Treasury security rates, $\Delta \widetilde{r}_{t}^{e}$ and $\widetilde{\varepsilon}_{t}$ denote the expected component and unanticipated components of a target change respectively, and $d_{t}^{C}=1$ if the target was changed at date $t$, and is 0 otherwise. Note that when the target is not changed, $d_{t}^{C}=0$, then $\Delta \widetilde{r}_{t}^{e}=-\widetilde{\varepsilon}_{t}$, so that $\beta_{2}$ is undetermined. Without loss of generality, we set $\beta_{2}=0$. 
Equation (19) is similar to equation (2) in Kuttner (2000) except that it allows the response of the surprise component of a target change to vary depending on whether the target actually changed or not. Another important departure with respect to Kuttner (2000) is that our estimates of the unanticipated component of target changes is done at a bi-weekly frequency rather than daily. This is important because it relates to the manner in which a surprise based on information that is two weeks old projects into today's rates. As we shall see, this has important implications in the manner in which we interpret the evidence. With the above disclaimer, it is natural to expect that the anticipated component of target changes will be already incorporated in current term rates and therefore the coefficient $\beta_{1}$ should be statistically insignificant. On the other hand, the unanticipated component of target changes carries new information to the market regarding the policy stance which should result in significant values for $\gamma_{1}$ and $\gamma_{2}$.

Figure 5 reports in graphical form the estimates of the parameter $\gamma_{1}$ in the left-hand panel and the estimates of $\gamma_{2}$ in the right-hand panel for the pre and post 1989 samples. The response of term rates to target surprises is quite strong during periods when the target was changed ( $\gamma_{1}$ estimates, left-hand panel), irrespective of the sample period, although the significance of the response decays with T-notes of increasing maturity, particularly after 1989. The response to target surprises during periods in which the target remained unchanged (displayed on the right-hand panel) is consistent with a form of announcement effect. The pre-1989 responses are statistically insignificant at all maturities suggesting that the market had little understanding on how the Fed operated in setting new targets. By con- 
trast, in the post-1989 period, the impact of the unanticipated component of target changes, monotonically increases with the maturity of the T-note and is statistically significant. This is consistent with the market having a better understanding of the target setting behavior and incorporating the information conveyed by surprise inaction into its long-term forecasts. We take these results as suggestive evidence that by regularizing and announcing policy decisions, the market is able to extract information on the policy stance both when the Fed changes interest rates unexpectedly as well as when it remains surprisingly inactive. The responses to the expected component of target changes are all insignificant at all maturities for both the pre and post 1989 samples in accordance to our predictions and for brevity, are not reported here. ${ }^{3}$

It is instructive to compare the results for the post-1989 with Kuttner's measure of target surprises. These results are displayed in figure 6. At first blush, these results appear unintuitive: although the responses to unanticipated target moves on days when the target was changed coincides rather closely with our own results, the response to anticipated target changes appears just as strong. By stacking these responses on top of each other, a natural explanation is that Kuttner's measure is based on the expectation based on information up to one day before the target change which does not appear to have been incorporated yet into term rates. Hence, the response of the 3-month bill to a target change (both anticipated and unanticipated components) is virtually 1 to 1 and monotonically decreases with the maturity of the T-note. Supporting this view, notice that the response of term

3 These results are available from the authors upon request. 
rates to surprise inaction (i.e., when an expected target change did not actually materialize) is insignificant at all maturities. The next section investigates in more detail the nature of some of the results reported above and advances some alternative explanations.

\subsection{Further Decompositions}

Unlike the forecasts one obtains from traditional linear time series models, the ACH/OP model allows us to investigate possible asymmetries in the response of term rates to Fed announcements. For example, we may conjecture that a target change of 50 basis points when a 25 basis points change was expected, has a different effect than a 25 basis point target change when the target change is unexpected. Although both of these events represent an equal surprise $\widetilde{\varepsilon}_{t}=0.25$, it is natural to assume that a $1 / 4 \%$ target change when none was expected may affect term rates differently because it carries the additional element of surprise "the target was changed."

A complete taxonomy of possible scenarios similar to the one presented above can be constructed as follows. Let $\widehat{x}_{t}$ denote the $\mathrm{ACH}$, one maintenance period ahead forecast of $x_{t}$, the indicator variable that takes the value of 1 if in period $t$ the target was changed and is 0 otherwise. Then, define the following dummy variables:

$$
\left\{\begin{array}{ccc}
d_{t}^{1,1}= & x_{t} \widehat{x}_{t} \\
d_{t}^{1,0}= & x_{t}\left(1-\widehat{x}_{t}\right) \\
d_{t}^{0,1}= & \left(1-x_{t}\right) \widehat{x}_{t} \\
d_{t}^{0,0}= & \left(1-x_{t}\right)\left(1-\widehat{x}_{t}\right)
\end{array}\right.
$$

so that $d_{t}^{1,1}=1$, if the target was changed in period $t$ and this was expected, 0 otherwise; 
$d_{t}^{1,0}=1$, if the target was changed and this was unexpected, 0 otherwise; $d_{t}^{0,1}=1$, if a target change was expected but none occurred, 0 otherwise; and $d_{t}^{0,0}=1$, if the target remained unchanged and this was expected. Note that the $i, j$ superscripts in $d_{t}^{i, j}$ take on the values of 0 or 1 with the $i^{t h}$ superscript indicating whether an actual target change took place $(i=1)$ or not $(i=0)$, and the $j^{t h}$ superscript indicates whether a target change was expected $(j=1)$ or not $(j=0)$. With these dummies, equation (19) can be respecified more completely as,

$$
\begin{aligned}
\Delta R_{t}= & {\left[\alpha_{1,1}+\beta_{1,1} \Delta \widetilde{r}_{t}^{e}+\gamma_{1,1} \widetilde{\varepsilon}_{t}\right] d_{t}^{1,1}+} \\
& {\left[\alpha_{1,0}+\beta_{1,0} \Delta \widetilde{r}_{t}^{e}+\gamma_{1,0} \widetilde{\varepsilon}_{t}\right] d_{t}^{1,0}+} \\
& {\left[\alpha_{0,1}+\beta_{0,1} \Delta \widetilde{r}_{t}^{e}+\gamma_{0,1} \widetilde{\varepsilon}_{t}\right] d_{t}^{0,1}+} \\
& {\left[\alpha_{0,0}+\beta_{0,0} \Delta \widetilde{r}_{t}^{e}+\gamma_{0,0} \widetilde{\varepsilon}_{t}\right] d_{t}^{0,0}+u_{t} }
\end{aligned}
$$

where the coefficients have been labeled with subscripts that correspond to the $i, j$ superscripts of the dummy variables. Note that when $d_{t}^{0,1}=1, \Delta \widetilde{r}_{t}^{e}=-\widetilde{\varepsilon}_{t}$ so that $\beta_{0,1}$ is undetermined. Similarly, when $d_{t}^{0,0}=1$, we set $\Delta \widetilde{r}_{t}^{e}=0$ (i.e., we denominate the difference between the forecast and 0 as $\left.\widetilde{\varepsilon}_{t}\right)$ so that $\beta_{0,0}$ is also undetermined. Without loss of generality, we set these coefficients to zero. Also note that although $d_{t}^{1,0}=1$ implies that a target change is not expected, $\Delta \widetilde{r}_{t}^{e}$ will in general be nonzero because in every period there is a nonzero probability of a target change. We include this variable in the corresponding part of the regression to prevent omitted variable bias but attach no specific interpretation to the corresponding coefficient, $\beta_{1,0}$. Similarly, note that when $d_{t}^{0,0}=1$, a target change is not expected and none actually occurs. However, the term $\widetilde{\varepsilon}_{t}$ will in general be nonzero for the 
reasons discussed above. Therefore, although we include this regressor to prevent omitted variable bias, we also attach no particular meaning to the coefficient $\gamma_{0,0}$.

The specification in equation (20) allows us to test several interesting hypotheses on the behavior of term rates:

1. $H_{0}^{1}: \beta_{1,1}=0$. When the target is changed, the expected component of target changes has no effect on term rates.

2. $H_{0}^{2}: \gamma_{1,1}=\gamma_{1,0}=0$. When the target is changed, the surprise component of target changes has no effect on term rates.

3. $H_{0}^{3}: \gamma_{1,1}=\gamma_{1,0}$. When the target is changed, the response of term rates to the surprise component of target changes does not depend on whether the action of changing the target was an expected event or not.

4. $H_{0}^{4}: \gamma_{0,1}=0$. When the target is not changed but the market expects a change, the magnitude of the surprise component affects term rates.

The outcome of these hypothesis tests will likely depend on the sample period that we consider. We have argued that prior to the 1989 break date, it was harder for the market to understand the timing with which the Fed operated on interest rates. Consequently, it may have been hard to read what actions had been taken and virtually impossible to create a substantive forecast on when to expect future Fed actions. Consequently, it may not be unreasonable to expect that $H_{0}^{1}$ and $H_{0}^{4}$ will fail to be rejected. By contrast, after the 1989 
break date, we have argued that Fed actions became noticeably more transparent (culminating in the 1994 policy of announcing target changes discussed in section 2). Therefore, we would still expect that $H_{0}^{1}$ would fail to be rejected to the extent that expected target changes are incorporated in term rates, although a better understanding of the Fed's operating procedures may result in a rejection of $H_{0}^{4}$. $H_{0}^{4}$ can also be seen as measuring the "nonannouncement" effect described in Roley and Sellon (1998), that is, measuring the market's reaction when it is surprised by the Fed's inactions.

Straightforward intuition suggests that we should reject $H_{0}^{2}$, and possibly reject $H_{0}^{3}$. Rejecting $H_{0}^{2}$ indicates that we expect the portion of a target change that is not expected to cause the market to revise its expectations on future values of the Fed funds rate and inflation and thus, to impact the term structure. $H_{0}^{3}$ is ambiguous: the event of a target change may carry an additional element of surprise that is reflected on term rates or it may not. Table 4 summarizes the results of these hypotheses tests while figure 7 displays the coefficient estimates of equation (20). As before, we also report the results obtained with Kuttner's procedures for the post-1989 sample to get a sense of the robustness of our findings.

The first hypothesis, $H_{0}^{1}: \beta_{1,1}=0$, fails to be rejected for the pre-1989 sample but not for the post-1989 sample. The value of the coefficient estimates for $\beta_{1,1}$ at each maturity is displayed on the top, left-hand panel of figure 7. A priori, we expected this hypothesis would fail to be rejected for both samples. However, the fact that it fails to be rejected during the first subsample but not during the second, underscores the argument that it was 
tricky to read what the Fed had done prior to 1989. Figure 7 also underscores that there are no substantial differences between our method of decomposing target changes and that advanced by Kuttner (2000). By contrast, the top, right-hand panel of figure 7 displays the coefficient estimates of $\gamma_{1,1}$, while the bottom left-hand panel displays the coefficient estimates of $\gamma_{1,0}$. These graphs do not reveal substantial differences across samples in the response of term rates to surprises during periods when the target was changed, irrespective of whether or not a target change was anticipated. This result is confirmed by the results in table 4 corresponding to the second hypothesis test, $H_{0}^{2}: \gamma_{1,1}=\gamma_{1,0}=0$, which is rejected in almost in every case, and the third hypothesis test, $H_{0}^{3}: \gamma_{1,1}=\gamma_{1,0}$, which in almost in every case, failed to be rejected. The main divergence in these general results takes place for the longest maturities (mostly the 10 and 30 year T-bonds).

The final hypothesis of interest, $H_{0}^{4}: \gamma_{0,1}=0$, corresponds to the non-announcement effect and is displayed graphically in the bottom, right-hand panel of figure 7. There appears to be no significant response to the Fed maintaining interest rates when a target change was expected during the pre-1989 sample. In fact, the coefficients turn negative for maturities over 1 year. On the other hand, we find a significant response of medium to long term maturities when measured with $\mathrm{ACH} / \mathrm{OP}$ forecasts. Although these responses appear to not be significant when measured with Kuttner's procedures, the significance levels are close to a $90 \%$ confidence level and the coefficient estimates behave in a similar fashion to the ACH/OP estimates. These results also support the argument that prior to 1989, the market had a hard time forming expectations on the timing of policy moves - there is no response 
to the non-announcement effect. The response of medium to long term maturity rates after 1989 is more in tune with the market understanding the schedule of FOMC meetings and becoming accustomed to the routine of the announcement.

\section{Conclusions}

This paper has explored the manner in which recent changes in the Fed's operating procedures may have altered the traditional monetary transmission mechanism. In particular, we concentrated on two key elements: The transmission of policy onto the overnight rate, and the transmission of movements in the overnight rate on to the term structure. The conventional view relies on the liquidity effect to explain how open market operations affect the overnight rate. However, in this paper we have argued that by publicly announcing the target for the Federal funds rate, the Fed poses a credible threat on to the Federal funds market, achieving a similar "liquidity effect" response. Consequently, the overnight rate may move to the new targeted level without requiring open market operations. Evidence from our VAR analysis is supportive of this view. We should remark that the Fed initially viewed the announcement effect negatively. Moreover, it seems clear that the motivation behind the initial public disclosure of a target change in 1994 was taken with extreme caution and was not initially viewed as a permanent change in operating procedures. However, it is also clear that the consequences of the announcement effect outlined in this paper support the usefulness of this practice since it appears that term rates are more responsive to policy movements. 
The monetary transmission mechanism requires of a second link to affect the real economy. This link typically relies on some type rational expectations mechanism that explains long-term rates as a weighted average of expected future short-term rates. In this paper, we presented a new methodology to discriminate the anticipated and surprise components of target changes, allowing us to measure their impact on term rates. Although the market of futures for federal funds provides a directly interpretable measure of surprise announcements, one can only go as far back as 1989. By contrast, we have shown that our methodology, while pairing well with this measure, can be used in periods that predate the formation of the futures market for federal funds and can be used to uncover the effect of target announcements on term rates. The data seems to partly validate our notions that, to the extent that the market incorporates expectations on futures rates into current term rates, only surprise changes in the target should influence the former. Moreover, we pursued a much more detailed characterization of the types of surprises the market faces in response to the Fed's behavior. In this respect, we find evidence that the market reacts to proactive monetary policy but also reacts vigorously to non-announcements at longer horizons.

In closing, the policy of publicly announcing interest rate targets has evolved into a proactive policy tool whose impact can be measured in the responsiveness of the overnight rate and terms rates to Fed policy moves. In addition, the rational expectations hypothesis seems to be well and alive: prices incorporate expectations on future overnight rates and in the long run, the policy stance determines the long term rate. 


\section{References}

Anderson, R. G. and Raasche, R. H. (2000), "Retail Sweep Programs and Bank Reserves, 1994-1999," Federal Reserve Bank of St. Louis, working paper 2000-023A.

Bernanke, B. S. and Mihov, I. (1998a), "Measuring Monetary Policy," Quarterly Journal of Economics, $113(3)$, 869-902.

Bernanke, B. S. and Mihov, I. (1998b), "The Liquidity Effect and Long-Run Neutrality," Carnegie-Rochester Conference Series on Public Policy, 49(0), 149-194.

Board of Governors of the Federal Reserve, (1991) FOMC Transcript, August 20, 1991.

Christiano, L. J. (1995), "Resolving the Liquidity Effect: Commentary," Federal Reserve Bank of St. Louis Review, 77(3), 55-61.

Clouse, J. A. (1994), "Recent Developments in Discount Window Policy," Federal Reserve Bulletin, November, 965-977.

Cook, T., and Hahn, T. (1989), "The Effect of Changes in the Federal Funds Rate Target on Market Interest Rates in the 1970s," Journal of Monetary Economics, 24, $33-51$.

Davutyan, N and Parke, W. R. (1995), "The Operations of the Bank of England, 1890-1908: A Dynamic Probit Approach," Journal of Money, Credit and Banking, 27, 1099-1112.

Dueker, M. (1998), "Conditional Heteroscedasticity in Qualitative Response Models of Time Series: A Gibbs Sampling Approach Applied to the Bank Prime Rate," working paper, Federal Reserve Bank of St. Louis.

Eichengreen, B., Watson, M. W., and Grossman, R. S. (1985), "Bank Rate Policy under the Interwar Gold Standard: A Dynamic Probit Model," Economic Journal 95, 725-745.

Federal Reserve Bank of New York, Market Groups. (1999), Domestic Open Market Operations During 1998.

Furfine, C. H. (2000), "Interbank Payments and the Daily Federal Funds Rate," Journal of Monetary Economics, Vol. 46, issue 2, 535-553.

Guthrie, G. and Wright, J. (2000), "Open Mouth Operations," Journal of Monetary Economics, Vol. 46, issue 2, 489-516.

Hamilton, J. D. (1996), "The Daily Market for Federal Funds," Journal of Political Economy, vol. 104, no. 11, 26-56. 
Hamilton, J. D. (1997), "Measuring the Liquidity Effect," American Economic Review, vol. 87 , no. 1, 80-97.

Hamilton, J. D. and Jordá, O. (2000), "A Model for the Federal Funds Rate Target," NBER working paper 784\%.

Hausman, J. A., Lo, A. W., and MacKinley, A. C. (1992), "An Ordered Probit Analysis of Transaction Stock Prices," Journal of Financial Economics, 31, 319-330.

Kuttner, K. (2000), "Monetary Policy Surprises and Interest Rates: Evidence from the Fed Funds Futures Market," Federal Reserve Bank of New York, manuscript.

Leeper, E. M., Sims, C. A. and Zha, T. (1996), "What Does Monetary Policy Do?" Brookings Papers on Economic Activity, 0(2), 1-63.

McCulloch, R. and Rossi, P. E. (1994), "An Exact Likelihood Analysis of the Multinomial Probit Model," Journal of Econometrics, 64, 207-240.

Meulendyke, A. M. (1998), U.S. Monetary Policy and Financial Markets, Federal Reserve Bank of New York.

Pagan, A. R. and Robertson, J. C. (1995), "Resolving the Liquidity Effect," Federal Reserve Bank of St. Louis Review, 77(3), 33-54.

Roley, V.V. and Sellon, G. H. (1998), "Market Reaction to Monetary Policy Nonannouncements," Federal Reserve Bank of Kansas City, Working Paper 98-06.

Rudebusch, G. D. (1995), "Federal Reserve Interest Rate Targeting, Rational Expectations and the Term Structure," Journal of Monetary Economics, 35(2), 245-274.

Strongin, S. (1995), "The Identification of Monetary Policy Disturbances. Explaining the Liquidity Puzzle," Journal of Monetary Economics 35, 463-497.

Thornton, D. L. (1998), "The Federal Reserve's Operating Procedure, Nonborrowed Reserves, Borrowed Reserves and the Liquidity Effect," Federal Reserve Bank of St. Louis, manuscript.

Thornton, D. L. (2000), "The Market Reaction to Changes in the Fed's Fund Rate Target: Has the Fed Used Open Mouth Operations?" Federal Reserve Bank of St. Louis, manuscript.

Woodford, M. (2000), "Monetary Policy in a World Without Money," NBER working paper 7853.

Wrase, J. M. (1998), "Is the Fed Being Swept Out of (Monetary) Control?" Federal Reserve Bank of Philadelphia Business Review, Nov-Dec, 3-12. 
Figure 1 - Required Reserves of Depository Institutions (Mil. \$)

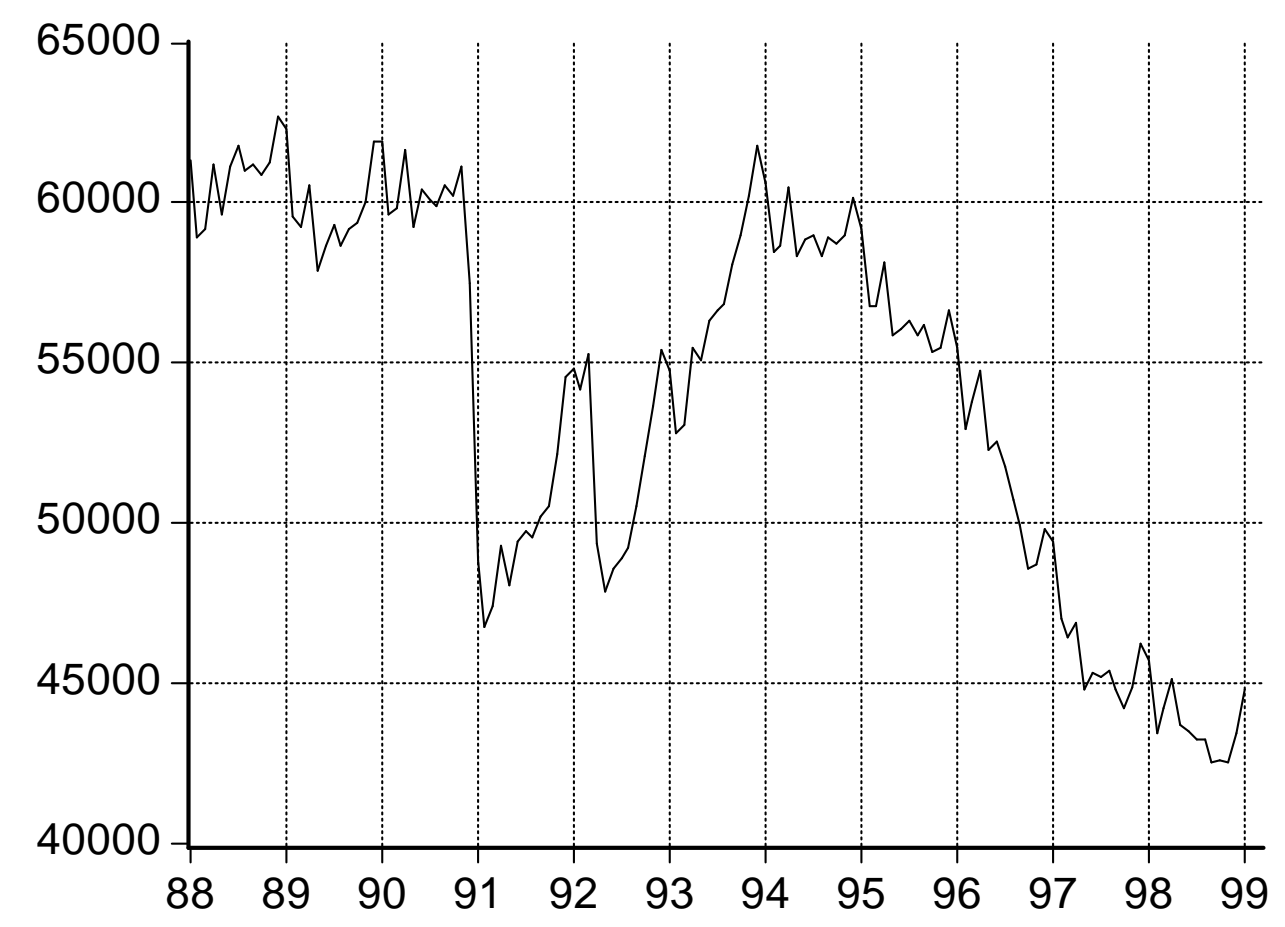

Figure 2 - Ratio of Non-Transaction to Transaction Deposits

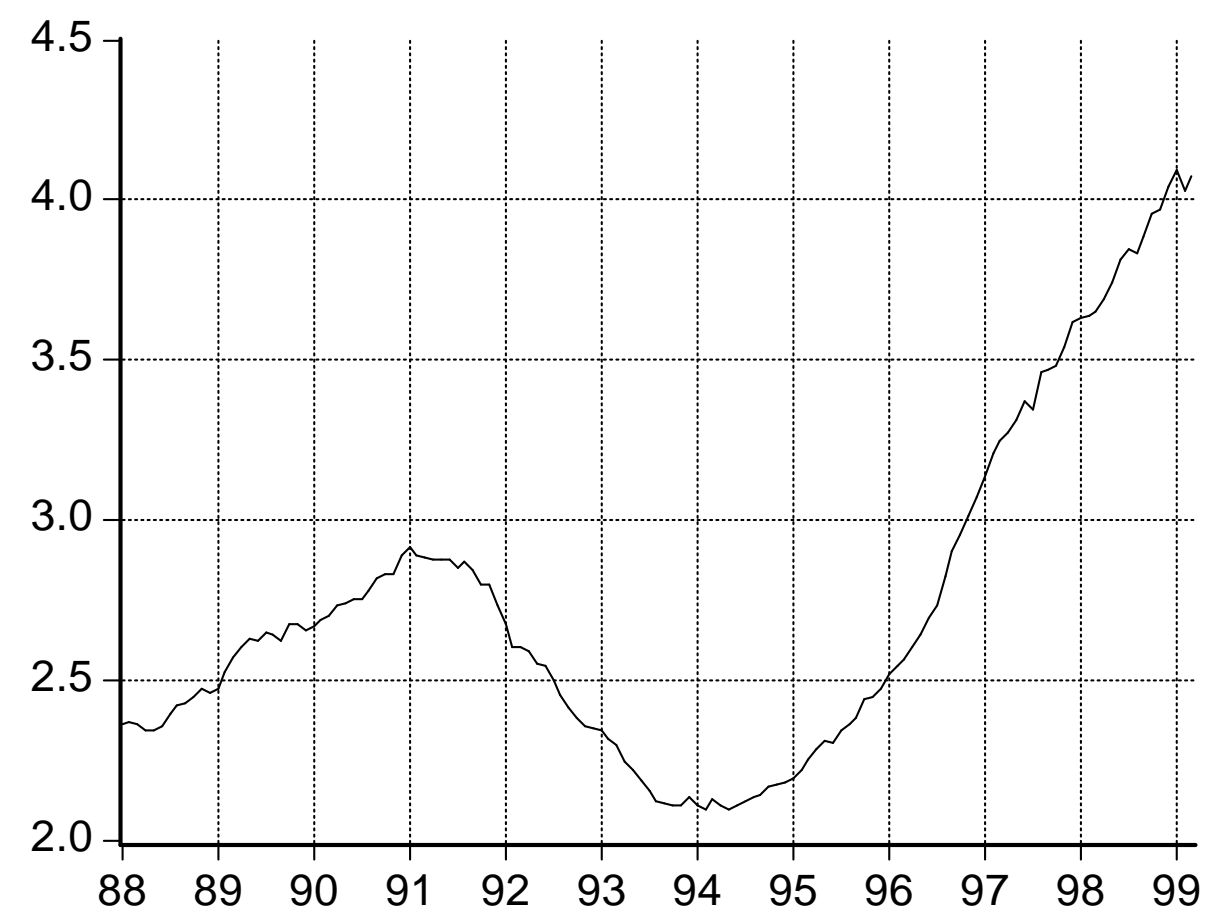


Figure 3. Daily Spread between the Prime Rate and the Federal Funds Rate Target

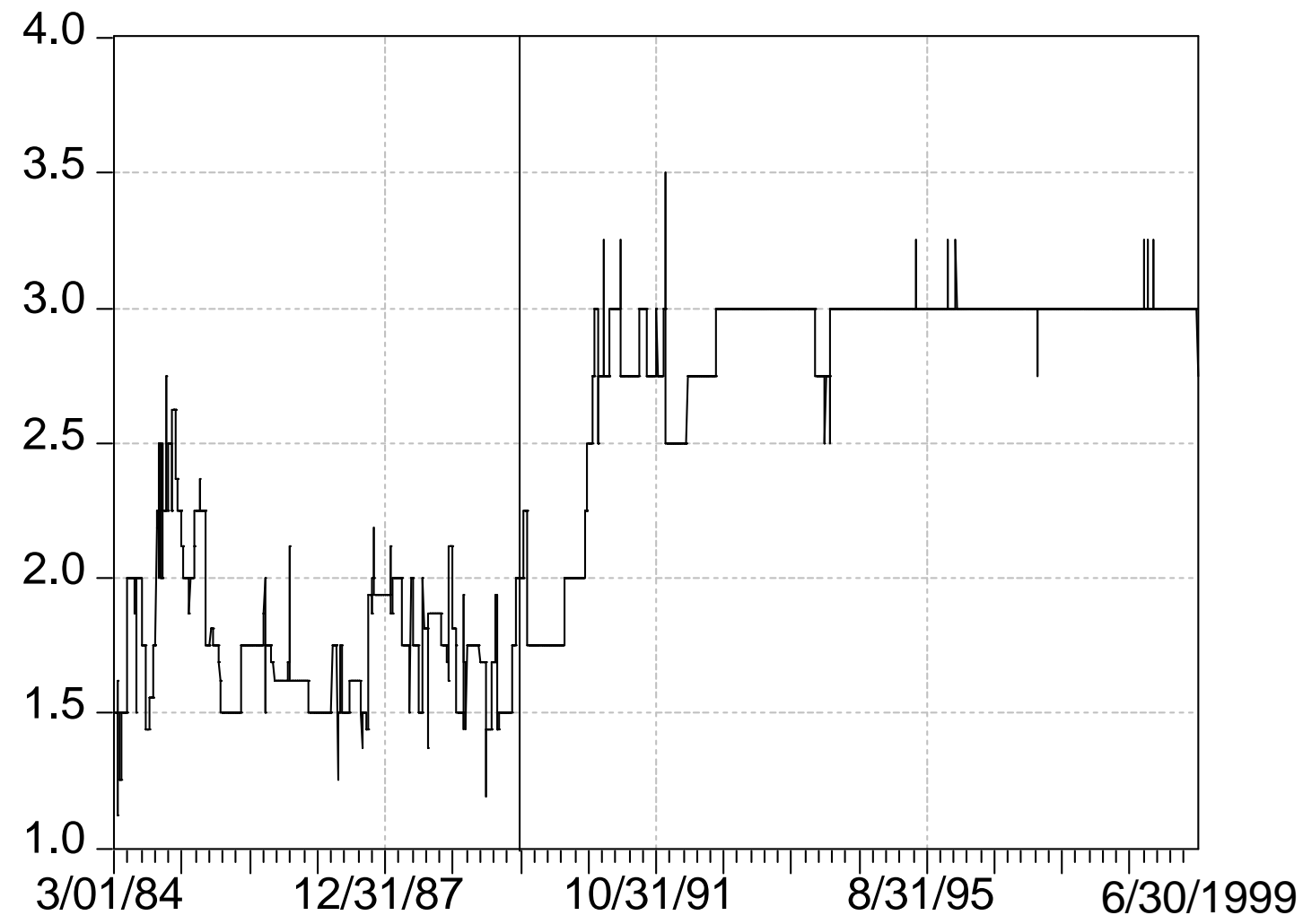

Note: The vertical line indicates the "Thanksgiving Effect" (November 22, 1989) 


\title{
Figure 4. Impulse Response Functions of the Reserves Market VAR Comparison of responses when periods with target changes are excluded
}

\author{
Reponse of FF to Shocks in NBRX \\ "The Liquidity Effect"
}

Pre - 1989 Sample

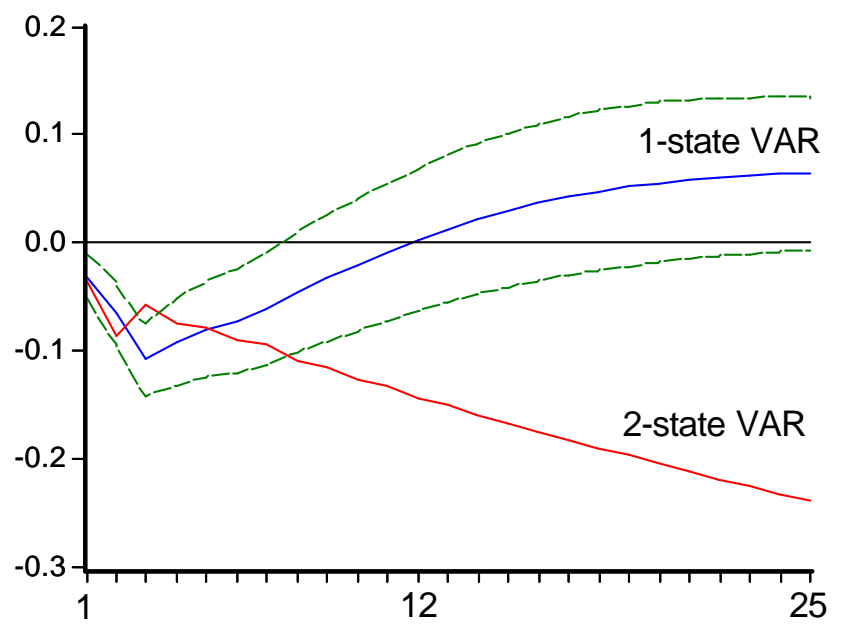

Post - 1989 Sample

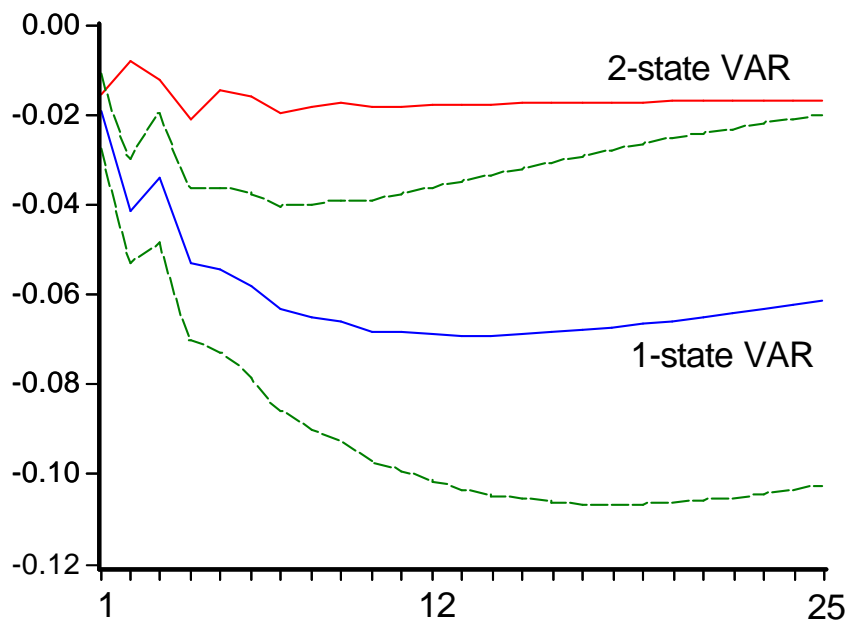

Response of NBRX to Shocks in NBRX

Pre - 1989 Sample

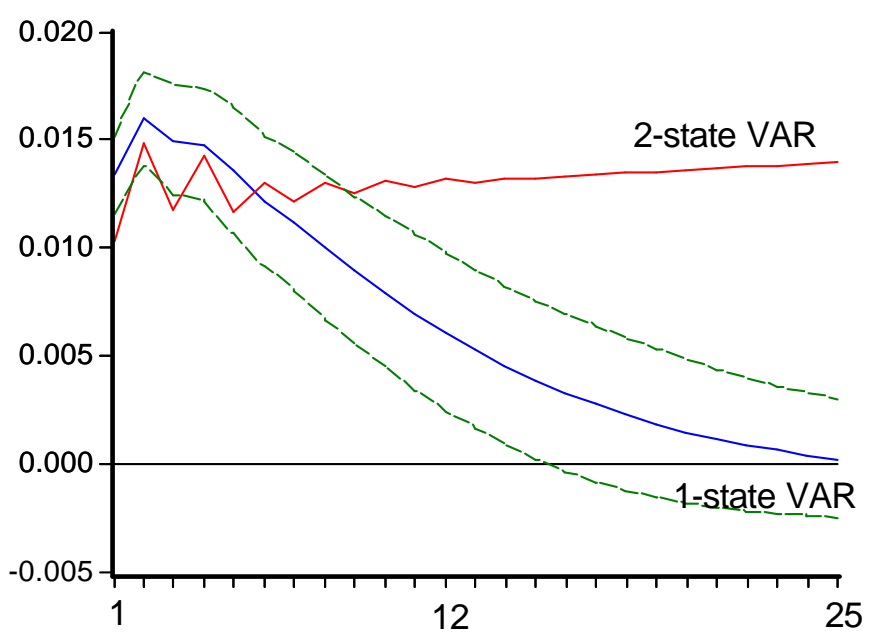

Post - 1989 Sample

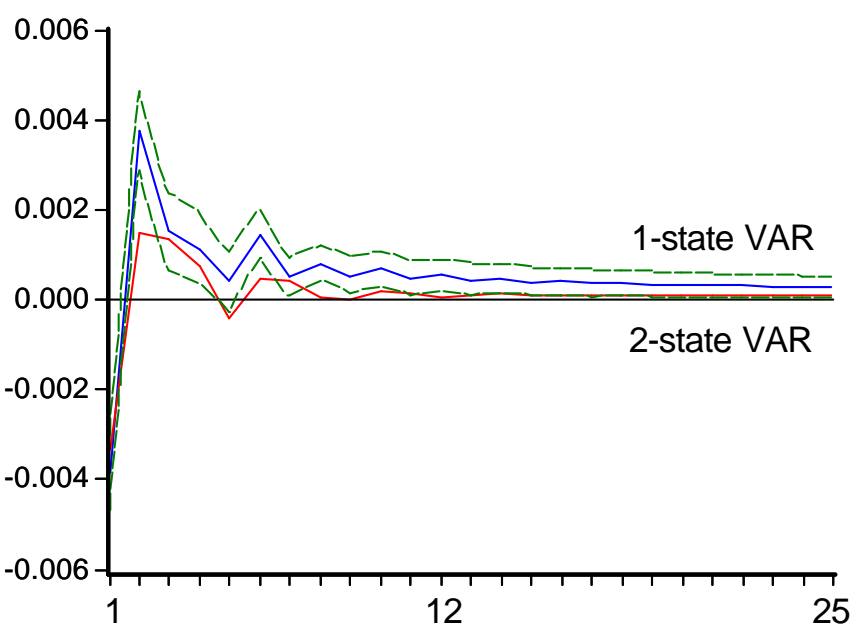

Notes:

- The VARs include the variables RRX, NBRX, and FF and the responses are calculated with the same Wold causal ordering.

- 1-state VAR: Refers to a VAR estimated with all the observations in the subsample.

- 2-state VAR: Refers to a VAR that excludes observations that correspond to changes in the Federal funds rate target.

- The error bands correspond to one standard deviation bands for VARs that include all the observations. 
Figure 5: Response of Treasury-note Rates to Unanticipated Changes in the Target

Response during Target Change Periods

Response during Periods when the Target is Unchanged
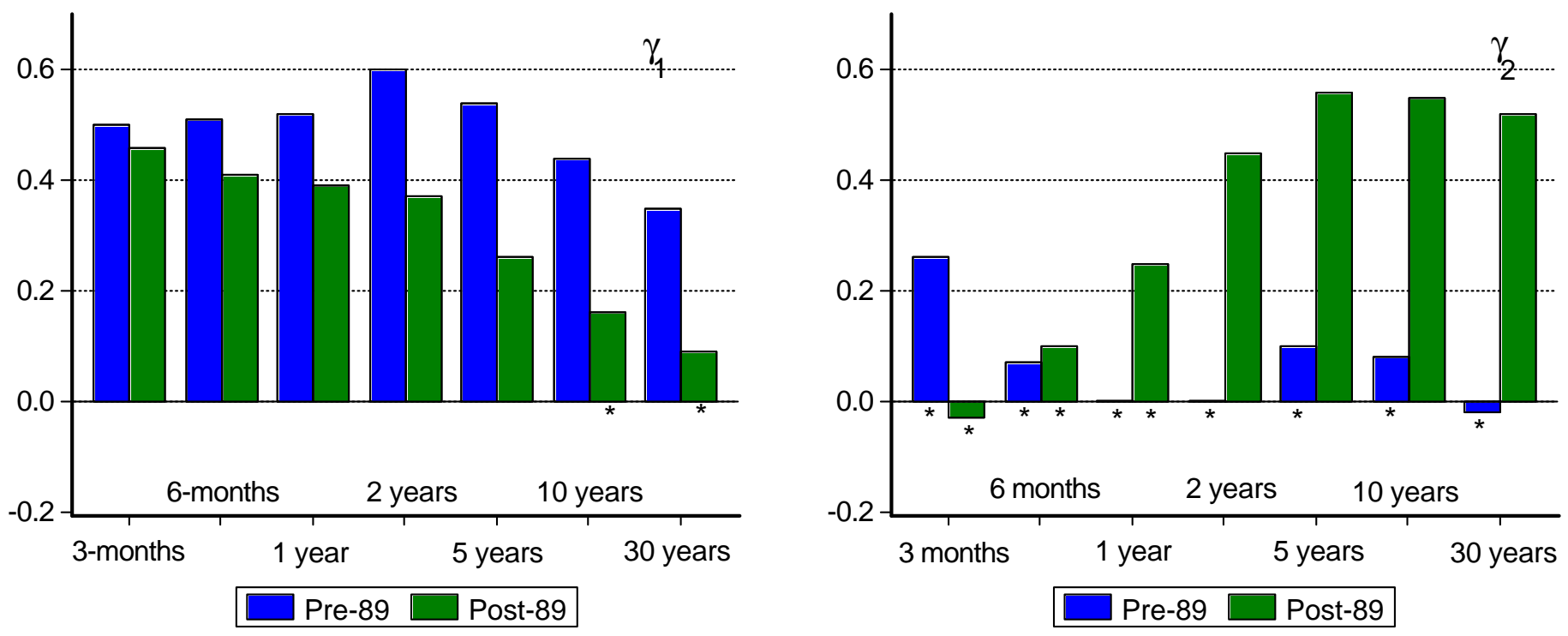

* denotes not significant at the conventional $95 \%$ confidence level 
Figure 6: Response of Treasury-note Rates to the Components of Target Changes/No Changes using Kuttner's (2000) Measure

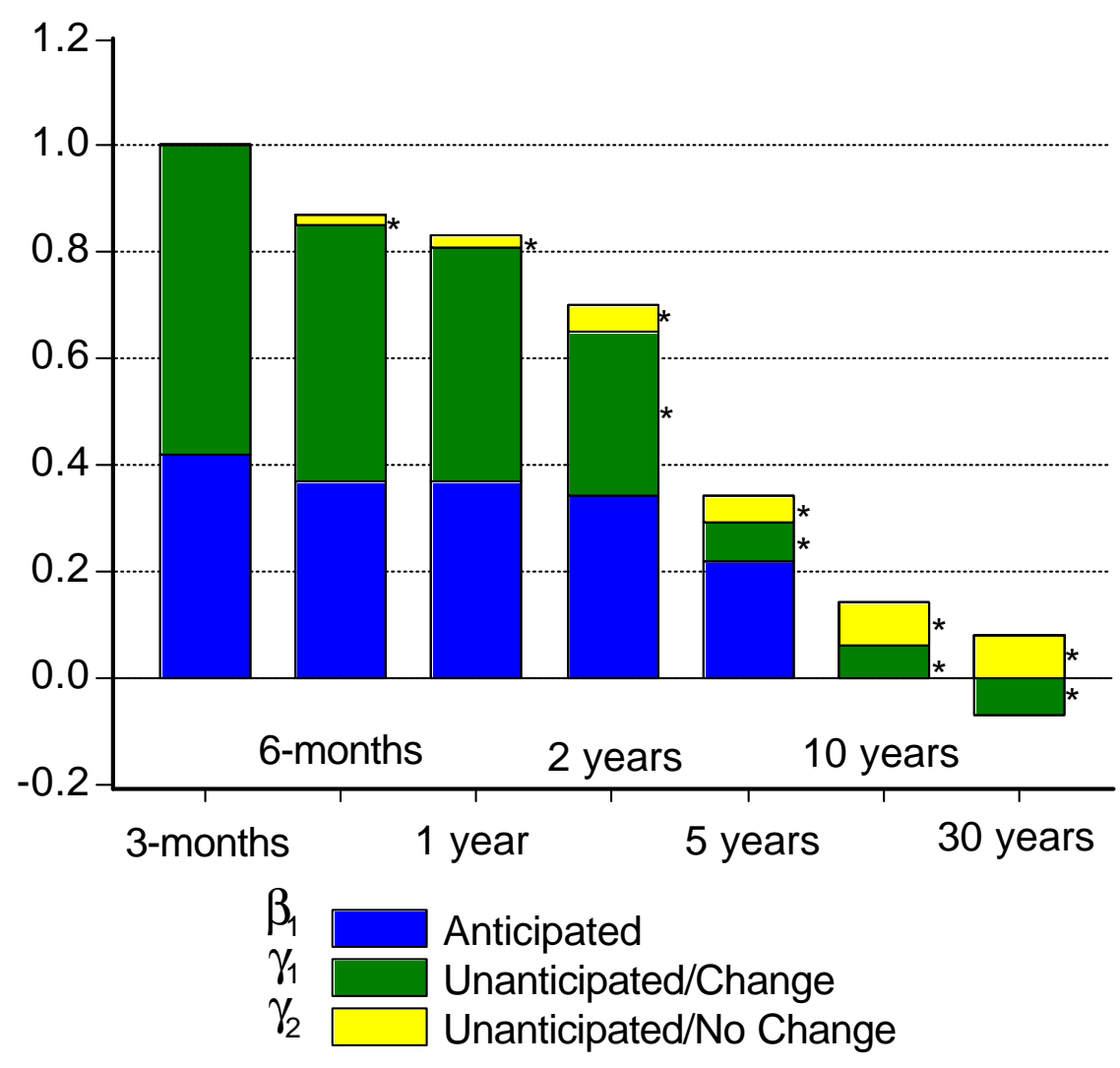

* denotes not significant at a $95 \%$ confidence level 


\section{Figure 7: Reponse of Treasury-note Rates to Different Elements of Surprise Target Changes/No Changes}

Response to the expected component of a target change that was expected

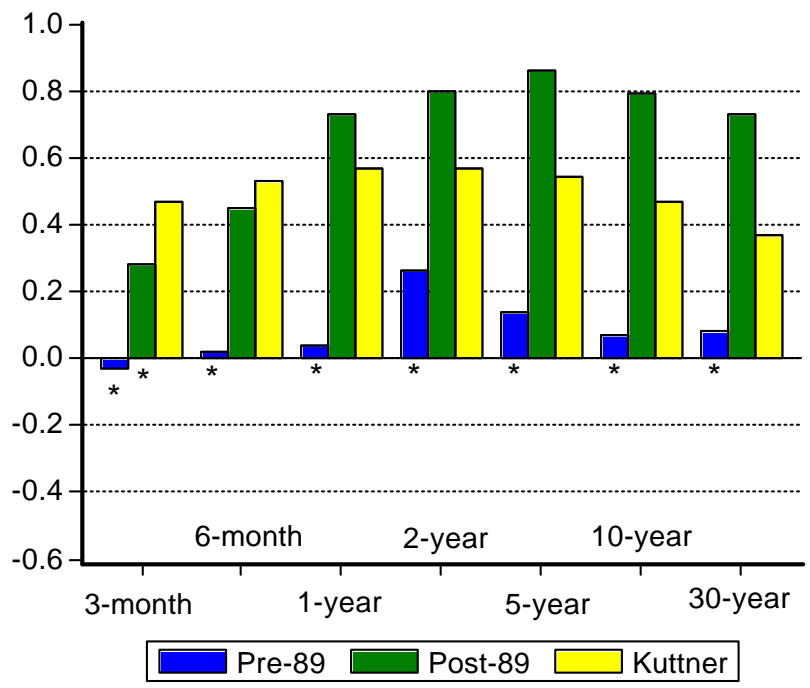

Reponse to the surprise component of a target change that was unexpected

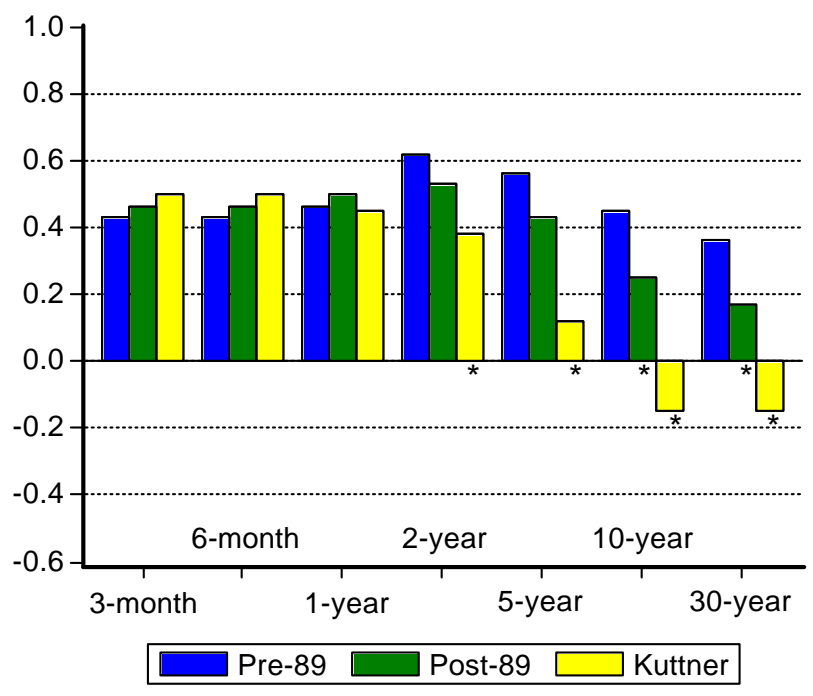

Response to the surprise component of a target change that was expected

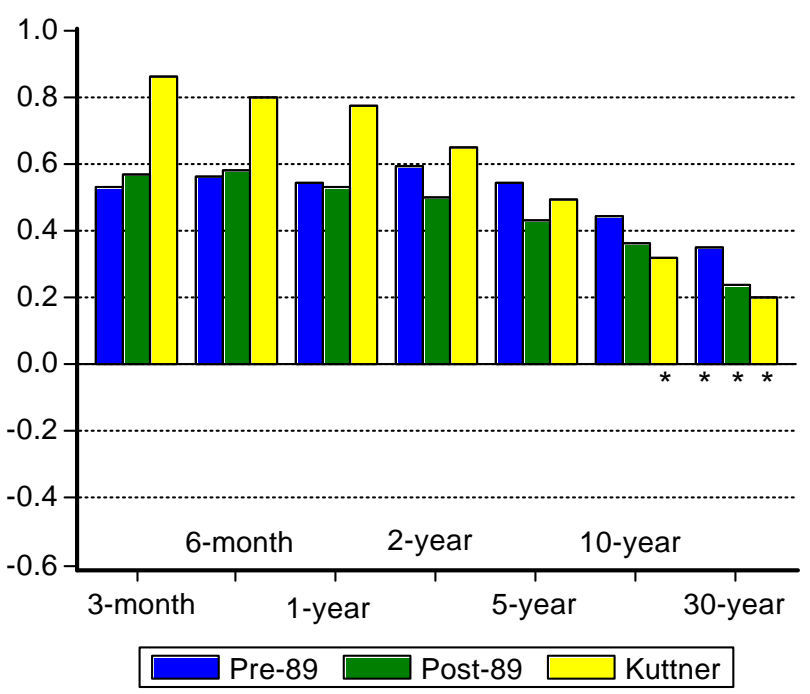

Response to the suprise component of unexpected inaction when a target change was expected

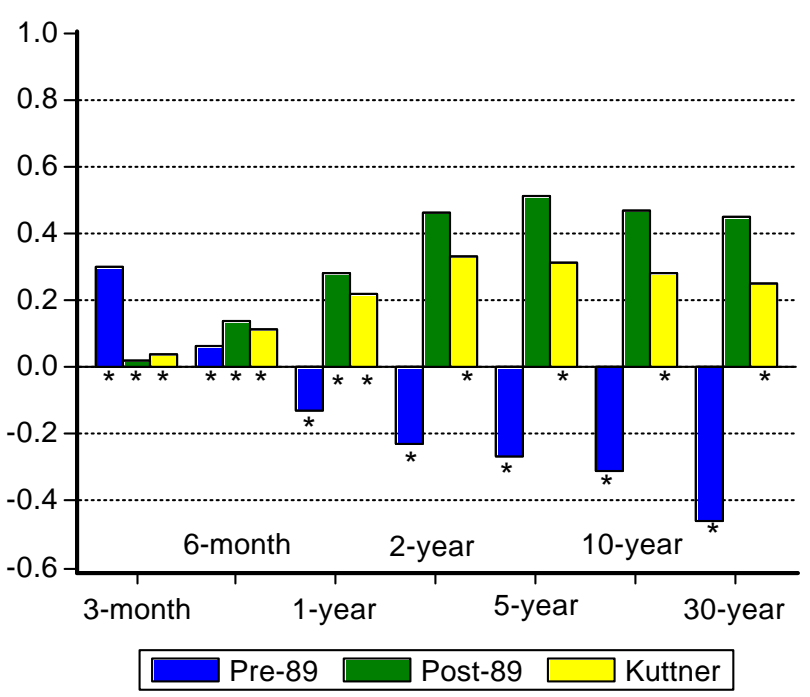

* Indicates not significant at the conventional 95\% confidence level 


\section{Table 1: Estimates for the ACH model}

Dependent Variable: Indicator Variable of Changes in the Federal Funds Rate Target Sample 1: 4/25/1984-11/1/1989

\begin{tabular}{clccc}
\hline \hline Parameter & Variable & Coefficient & Std. Err. & t-statistic \\
\hline$\delta_{1}$ & Constant $_{2}$ & 1.32 & 0.41 & 3.22 \\
$\delta_{2}$ & FOMC $_{\mathrm{t}}$ & -0.95 & 0.40 & -2.39 \\
$\delta_{3}$ & FOMC $_{\mathrm{t}-1}$ & -0.67 & 0.35 & -1.92 \\
$\delta_{4}$ & Inflation Rate $_{\mathrm{t}-1}$ & -5.79 & 2.47 & -2.34 \\
$\theta$ & $u_{1, t-1}$ & 0.07 & 0.05 & 1.43 \\
$\beta$ & $\lambda_{1, t-1}$ & 0.75 & 0.11 & 6.91 \\
\hline Mean Log-Likelihood: -90.91 & & & Sensitivity: 0.74 \\
Persistence: $\theta+\beta=0.82$ & & & Specificity: 0.55 \\
\hline \hline
\end{tabular}

Sample 2: 11/15/1989-3/24/1999

\begin{tabular}{clccc}
\hline \hline Parameter & Variable & Coefficient & Std. Err. & t-statistic \\
\hline$\delta_{1}$ & Constant & 2.29 & 0.54 & 4.21 \\
$\delta_{2}$ & FOMC $_{\mathrm{t}}$ & -1.55 & 0.43 & -3.56 \\
$\delta_{3}$ & $\mid$ FFR-TB6 $_{\mathrm{t}-1}$ & -2.22 & 0.50 & -4.46 \\
$\delta_{4}$ & Duration PR $_{\mathrm{t}-1}$ & -0.04 & 0.02 & -1.81 \\
$\theta$ & $u_{1, t-1}$ & 0.03 & 0.03 & 0.84 \\
$\beta$ & $\lambda_{1, t-1}$ & 0.51 & 0.13 & 3.80 \\
\hline Mean Log-Likelihood :-86.97 & & & Sensitivity: 0.54 \\
Persistence: $\theta+\beta=0.54$ & & & Specificity: 0.67 \\
\hline \hline
\end{tabular}

Data Frequency: Bi-weekly, maintenance period. 
Table 2: Estimates for the Ordered Probit Model

Sample 1: 4/11/1984-11/1/1989

\begin{tabular}{ccccc}
\hline \hline Variable & Coefficient & Std. Error & z-Statistic & Prob. \\
\hline$\Delta$ FFRT $_{\mathrm{t}-1}$ & 1.22 & 0.58 & 2.10 & 0.04 \\
BRX $_{\mathrm{t}-1}$ & -5.79 & 2.24 & -2.59 & 0.01 \\
FFR-TB6 $_{\mathrm{t}-1}$ & -1.35 & 0.51 & -2.66 & 0.01 \\
SFFRTDR $_{\mathrm{t}-1}$ & 0.55 & 0.27 & 2.05 & 0.04 \\
& & Limit Points & & \\
& -1.97 & 0.41 & -4.87 & 0.00 \\
LIMIT_2 & -0.89 & 0.35 & -2.52 & 0.01 \\
LIMIT_3 & -0.48 & 0.34 & -1.40 & 0.16 \\
LIMIT_4 & 1.19 & 0.38 & 3.15 & 0.00 \\
LIMIT_5 & -71.75672 & & & \\
\hline Log likelihood & -71.96 &
\end{tabular}

Sample 2: 11/15/1989-3/24/1999

\begin{tabular}{ccccc}
\hline \hline Variable & Coefficient & Std. Error & z-Statistic & Prob. \\
\hline FFR-TB6 $_{\mathrm{t}-1}$ & -3.98 & 1.45 & -2.75 & 0.01 \\
SFFRTDR $_{\mathrm{t}-1}$ & 6.07 & 2.02 & 3.00 & 0.00 \\
SPRFFRT $_{\mathrm{t}-1}$ & 5.22 & 1.89 & 2.76 & 0.01 \\
DFFRTDR $_{\mathrm{t}-1}$ & 0.03 & 0.01 & 2.10 & 0.04 \\
& & Limit Points & & \\
& & 5.55 & 2.55 & 0.01 \\
LIMIT_2 & 14.15 & 5.97 & 2.93 & 0.00 \\
LIMIT_4 & 17.50 & 7.54 & 2.88 & 0.00 \\
LIMIT_5 & 21.75 & & & \\
\hline Log likelihood & -15.14 &
\end{tabular}

$$
y_{i}=\left\{\begin{array}{rrr}
-0.50 & \text { if } & \Delta \tilde{r}_{i}<-0.375 \\
-0.25 & \text { if } & -0.375 \leq \Delta \tilde{r}_{i}<-0.0625 \\
0.00 & \text { if } & -0.0625 \leq \Delta \tilde{r}_{i}<0.0625 \\
0.25 & \text { if } & 0.0625 \leq \Delta \tilde{r}_{i}<0.375 \\
0.50 & \text { if } & 0.375 \leq \Delta \tilde{r}_{i}
\end{array}\right.
$$

Note: Sample 2 contains no changes smaller than 25 basis points in absolute value. Thus, the category, "change of size 0 " is dropped and therefore only 3 limit points are necessary. 


\section{Table 3: Estimates for the Logit Model based on Kuttner's Federal Funds Futures Measure}

\begin{tabular}{ccccc}
\hline \hline Variable & Coefficient & Std. Error & z-Statistic & Prob. \\
\hline C & -3.27 & 0.36 & -9.01 & 0.00 \\
|Anticipated & 10.36 & 1.69 & 6.14 & 0.00 \\
\hline Log likelihood & -65.13 & & & Sensitivity $=0.88$ \\
& & & Specificity $=0.80$ \\
\hline \hline
\end{tabular}

\section{Notes:}

1) Dependent Variable: Binary indicator of changes in the target.

2) Anticipated $\mid$ is the absolute value of expected changes in the Federal funds rate target calculated from the Federal funds futures market using Kuttner's methodology.

3) The sample period includes 36 changes in the Federal funds rate target from 4/5/1989 through 1/28/1998. 
Table 4: Hypothesis tests on the effects of market expectations on term rates

The tests are based on the equation (20) in the text, reproduced here for convenience:

$$
\begin{aligned}
& \Delta R_{t}=\left[\alpha_{1,1}+\beta_{1,1} \Delta \tilde{r}_{t}^{e}+\gamma_{1,1} \widetilde{\varepsilon}_{t}\right] d_{t}^{1,1}+\left[\alpha_{1,0}+\beta_{1,0} \Delta \tilde{r}_{t}^{e}+\gamma_{1,0} \widetilde{\varepsilon}_{t}\right] d_{t}^{1,0}+ \\
& {\left[\alpha_{0,1}+\beta_{0,1} \Delta \tilde{r}_{t}^{e}+\gamma_{0,1} \widetilde{\varepsilon}_{t}\right] d_{t}^{0,1}+\left[\alpha_{0,0}+\beta_{0,0} \Delta \tilde{r}_{t}^{e}+\gamma_{0,0} \widetilde{\varepsilon}_{t}\right] d_{t}^{0,0}+u_{t}}
\end{aligned}
$$

1. $H_{0}^{1}: \beta_{1,1}=0$

2. $H_{0}^{2}: \gamma_{1,1}=\gamma_{1,0}=0$

3. $H_{0}^{3}: \gamma_{1,1}=\gamma_{1,0}$

4. $H_{0}^{4}: \gamma_{0,1}=0$

Pre-1989 Sample (ACH/OP Forecasts)

p-value

\begin{tabular}{l|ccccccc}
\hline \multicolumn{1}{c}{ Hypothesis } & 3-month & 6-month & 1-year & 2-year & 5-year & 10-year & 30-year \\
\hline \hline$H_{0}^{1}: \beta_{1,1}=0$ & .92 & .96 & .93 & .62 & .78 & .88 & .86 \\
$H_{0}^{2}: \gamma_{1,1}=\gamma_{1,0}=0$ & .00 & .00 & .00 & .00 & .00 & .00 & .01 \\
$H_{0}^{3}: \gamma_{1,1}=\gamma_{1,0}$ & .50 & .49 & .66 & .90 & .92 & .97 & .97 \\
$H_{0}^{4}: \gamma_{0,1}=0$ & .52 & .89 & .78 & .61 & .58 & .49 & .26 \\
\hline \hline
\end{tabular}

Post-1989 Sample (ACH/OP Forecasts)

p-value

\begin{tabular}{l|ccccccc}
\hline \multicolumn{1}{c}{ Hypothesis } & 3-month & 6-month & 1-year & 2-year & 5-year & 10-year & 30-year \\
\hline \hline$H_{0}^{1}: \beta_{1,1}=0$ & .20 & .04 & .00 & .00 & .00 & .01 & .01 \\
$H_{0}^{2}: \gamma_{1,1}=\gamma_{1,0}=0$ & .00 & .00 & .00 & .00 & .00 & .01 & .07 \\
$H_{0}^{3}: \gamma_{1,1}=\gamma_{1,0}$ & .52 & .41 & .83 & .83 & .97 & .56 & .69 \\
$H_{0}^{4}: \gamma_{0,1}=0$ & .91 & .35 & .07 & .03 & .04 & .05 & .06 \\
\hline \hline
\end{tabular}

Post-1989 Sample (Kuttner Forecasts)

p-value

\begin{tabular}{l|ccccccc}
\hline \multicolumn{1}{c}{ Hypothesis } & 3-month & 6-month & 1-year & 2-year & 5-year & 10-year & 30-year \\
\hline \hline$H_{0}^{1}: \beta_{1,1}=0$ & .00 & .00 & .00 & .00 & .00 & .00 & .00 \\
$H_{0}^{2}: \gamma_{1,1}=\gamma_{1,0}=0$ & .00 & .00 & .00 & .00 & .07 & .22 & .34 \\
$H_{0}^{3}: \gamma_{1,1}=\gamma_{1,0}$ & .02 & .11 & .10 & .34 & .26 & .09 & .16 \\
$H_{0}^{4}: \gamma_{0,1}=0$ & .63 & .22 & .10 & .10 & .14 & .13 & .11 \\
\hline \hline
\end{tabular}

